\title{
Editorial
}

\section{Autophagic cell death RIPs into tumors}

\author{
A Oberst ${ }^{*, 1}$ \\ Cell Death and Differentiation (2013) 20, 1131-1132; doi:10.1038/cdd.2013.89
}

\section{Autophagic cell death RIPs into tumors}

The idea of 'autophagic cell death' has been the subject of some controversy, much of it focused on links between autophagy and apoptosis. In this issue of $C D D$, Basit et al. ${ }^{1}$ report that the chemotherapeutic drug Obatoclax triggers autophagy-dependent cell death, but that this death is RIPK-dependent necroptosis, not apoptosis.

Autophagy (also called macroautophagy) is a ubiquitous and evolutionarily conserved process of cellular 'self-eating.' During autophagy, multiple cellular signaling cascades converge on the ATG proteins, which mediate the formation of double-membraned vesicles around cytosolic components or organelles. These autophagosomes then fuse with lysosomes, leading to the breakdown and recycling of their contents. Autophagy is thus a key component of the cellular stress response, allowing the removal of damaged organelles or catabolism of cellular components to generate energy during starvation. Apoptosis is another form of cellular stress response, and in many cases apoptotic cell death is accompanied by upregulation of autophagy. While some studies initially interpreted this as evidence for autophagy directly triggering apoptosis, it is now generally accepted that autophagy is a cytoprotective mechanism that promotes cell survival, and that apoptosis ensues when this protection fails. ${ }^{2}$ The idea of 'autophagic cell death'-meaning programmed cell death that requires autophagy-has fallen out of favor.

Nonetheless, mechanistic ties between autophagy and apoptosis do exist. Notably, the antiapoptotic Bcl-2 family proteins can bind to and sequester the key autophagic activator beclin-1. ${ }^{3}$ Furthermore, several studies have reported interactions between the ATG5-12 complexes and the extrinsic apoptotic adapter protein FADD; these interactions recruit FADD to autophagosomes. ${ }^{4-6}$

In this issue, Basit et al. ${ }^{1}$ provide convincing evidence for autophagy-driven cell death mediated by these interactions. They show that the treatment of rhabdomyosarcoma cells with the pan-Bcl-2 family inhibitor Obatoclax (also called GX15-070) leads to massive autophagy and potent cell death; surprisingly however, they demonstrate that this cell death is not caspasedependent apoptosis, but rather receptor interacting protein kinase-1 (RIPK1) and RIPK3-dependent necroptosis.

Necroptosis is a form of caspase-independent cell death distinct from apoptosis; necroptosis depends on the activation of RIPK1 and RIPK3, though the mechanisms by which these enzymes lead to cell death remain to be elucidated. As its name implies, RIPK1 can be activated by cell surface receptors, notably the TNF-family death receptors and the TRIF-dependent toll-like receptors, TLR3 and TLR4. ${ }^{7}$ Following its activation, RIPK1 forms a cytosolic complex containing RIPK3, which can promote RIPK3 activation and necroptosis; however, under normal cellular conditions this complex also recruits the proapoptotic protease caspase- 8 and its paralog $\mathrm{CFLIP}_{\mathrm{L}}$, which suppress necroptosis and allow cellular survival $^{8}$ (Figure 1a). Here, Basit et al. ${ }^{1}$ demonstrate that upon induction of autophagy by Obatoclax, a similar complex is recruited to the autophagosome in an ATG5-dependent manner. Strikingly, this recruitment leads to potent RIPK3 activation and necroptotic cell death; this study therefore resurrects the concept of 'autophagic cell death', though in the guise of necroptosis rather than apoptosis (Figure 1b).

This work raises several questions. First, while the authors convincingly demonstrate that Obatoclax-driven autophagy is required to trigger necroptosis, the mechanism by which this putative Bcl-2 inhibitor promotes autophagy is not well understood. Indeed, the authors demonstrate that while another Bcl-2 inhibitor, ABT737, induced potent apoptosis without hallmarks of autophagy, Obatoclax fails to drive apoptosis while promoting a massive autophagic response. Earlier studies using bax/bak-deficient cells have demonstrated that while ABT737 is indeed a specific inhibitor of the Bcl-2 family, Obatoclax has notable off-target effects. ${ }^{9}$ While this observation does not affect the authors' demonstration of autophagosome-dependent necroptosis, it does raise questions about the cellular target of Obatoclax; for example, it would be useful to know whether other inducers of autophagy, such as nutrient starvation or rapamycin, could similarly promote necroptosis in these cell lines.

Another striking feature of the autophagy-induced necroptosis described by Basit et al. ${ }^{1}$ is the lack of involvement of caspase-8. During normal receptor signaling, caspase-8 recruitment to the RIPK1/RIPK3 complex limits RIPK activation and thereby suppresses necroptosis (Figure1a); most studies of necroptosis rely on chemical inhibition or genetic ablation of caspase- 8 to promote RIPK3 activation. The study by Basit et al. ${ }^{1}$ joins several other papers in proposing that intracellular membranes can also act as assembly platforms for these receptorassociated complexes. For example, several studies have observed FADD-dependent recruitment of caspase-8 to autophagosomes. $^{4-6}$ Furthermore, autophagosome-dependent recruitment of caspase-8 was shown to suppress

\footnotetext{
${ }^{1}$ Department of Immunology, University of Washington, Seattle, WA, USA

*Corresponding author: A Oberst, Department of Immunology, Campus Box 358059, 750 Republican St. Seattle, WA 98109, USA. Tel: (206) 221 7316; Fax: (206) 543 1013; E-mail: Oberst@uw.edu
} 


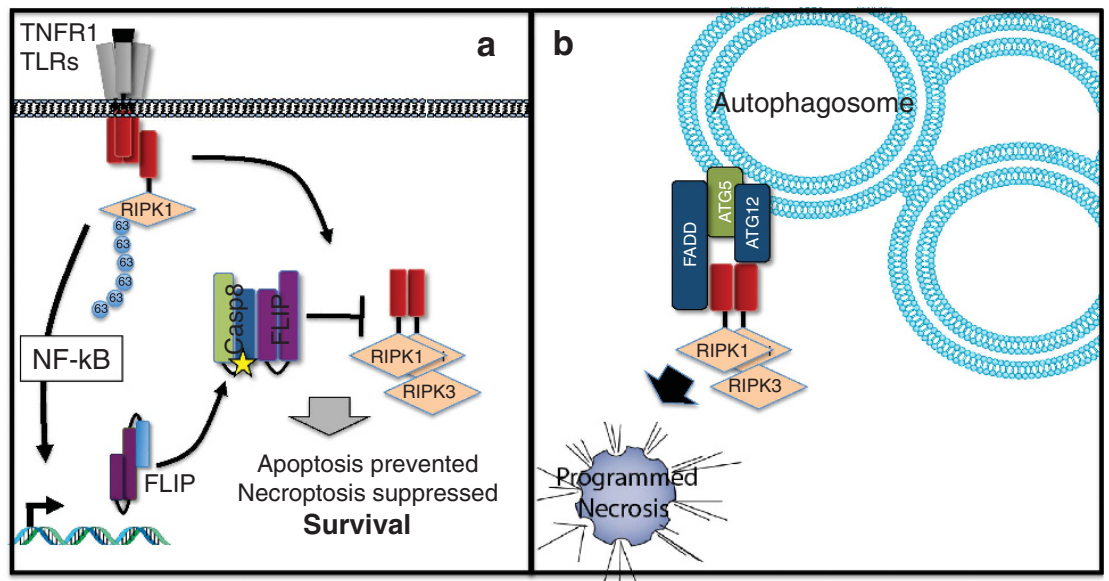

Figure 1 Receptor- and autophagosome-driven necroptosis. (a) Control of RIPK activation and cell death during normal receptor signaling. NF- $k B$-dependent $c F L I P L$ expression engages caspase-8 to suppress necroptosis without triggering apoptosis. (b) In this issue, Basit et al. ${ }^{1}$ show that induction of autophagy by the drug Obatoclax leads to the recruitment of RIPK1 and RIPK3, along with the adapter protein FADD, to autophagosomes. This in turn leads to RIPK3 activation and necroptosis. Notably, in this setting caspase-8 does not suppress RIPK3 activation

RIPK3-dependent necroptosis and allow successful T-cell proliferation, ${ }^{4}$ though subsequent genetic studies challenged this conclusion. ${ }^{10}$ Similarly, another study showed that caspase-8 inhibition promoted autophagy-dependent activation of necroptosis in $L 929$ cells. ${ }^{11}$ These observations are consistent with the idea that autophagic membranes can recapitulate the protein complexes and cell death pathways normally activated by receptors, and that in both cases caspase-8 recruitment keeps necroptosis in check.

It is therefore surprising that Basit et al. ${ }^{1}$ observe recruitment of FADD to Obatoclax-induced autophagosomes, but do not observe caspase-8 in these complexes; rather, autophagosome-associated FADD seems to directly recruit RIPK1 and RIPK3, and importantly caspase inhibition is not required for necroptosis in this setting. Tellingly, another recent report of autophagosome-induced necroptosis drew similar conclusions; this study, by Bray et al., found that simultaneously inducing autophagy using an mTOR inhibitor and preventing autophagosome maturation using chloroquine led to autophaogosome accumulation and RIPK3-dependent death in renal cell carcinoma lines. In neither case was caspase inhibition necessary; but why not?

A possible answer may lie in the observation that triggering necroptosis via drug-induced autophagy does not lead to the same transcriptional signature as receptor-mediated RIPK1 activation. Both death receptor and TLR signaling activate the $\mathrm{NF}-\kappa \mathrm{B}$ transcriptional program, which upregulates survival factors including the caspase-8 paralog $c F L I P_{L}$. CFLIP $P_{L}$ forms a catalytically active heterodimer with caspase-8, and this heterodimeric species is required for suppression of necroptosis. ${ }^{8}$ Importantly, NF- $\kappa \mathrm{B}$ signaling is engaged in both proliferating T cells and L929 cells (the latter due to constitutive autocrine TNF signaling ${ }^{12}$ ), and in these systems it appears that autophagosome-dependent RIPK activation is indeed suppressed by caspase-8. It may therefore be the case that drug-induced autophagy is potently pronecroptotic because it allows activation of the RIPKs without the prosurvival transcriptional response associated with engaging these pathways via cell surface receptors (Figure 1b).
A final question raised by this work, as by the discovery of genetically encoded necrosis-like cell death in general: What is the effect of engaging these pathways in vivo, and in particular in the setting of a tumor? Evasion of immune responses is a hallmark of deadly malignancies, and apoptosis is generally thought to promote immune tolerance. ${ }^{13}$ Necroptosis is believed to release damage-associated activators of innate immunity that are contained or neutralized by apoptosis; furthermore, increased autophagy in dying cells promotes the cross-presentation of dead cell-derived antigens to CD8 $+\mathrm{T}$ cells. ${ }^{14}$ These observations would imply that induction of autophagy-associated necroptosis in tumors might trigger a beneficial anti-tumor immune response. On the other hand, multiple lines of evidence support the notion that inflammatory cytokines produced by tumor-associated immune cells can act on non-tumor tissues to promote angiogenesis and neoplastic invasion, as well as driving proliferation of the tumor itself; ${ }^{15}$ inflammatory cell death in tumors would reinforce these detrimental effects.

As the study of cell death expands beyond apoptosis, we must increasingly consider not only whether a cell dies, but how that cell dies, and how that death influences surrounding tissues and global immune responses. By identifying Obatoclax as an inducer of autophagy-associated necroptosis, Basit et al. ${ }^{1}$ have provided a tool for these efforts; we must now figure out how to use it.

\footnotetext{
1. Basit F, Cristofanon S, Fulda S. Cell Death Differ 2013; 20: 1161-1173.

2. Kroemer G, Levine B. Nat Rev Mol Cell Biol 2008; 9: 1004.

3. Pattingre $S$ et al. Cell 2005; 122: 927.

4. Bell BD et al. Proc Natl Acad Sci USA 2008; 105: 16677.

5. Pyo JO et al. J Biol Chem 2005; 280: 20722.

6. Young MM et al. J Biol Chem 2012; 287: 12455.

7. Vandenabeele $\mathrm{P}$ et al. Nat Rev Mol Cell Biol 2010; 11: 700.

8. Oberst A et al. Nature 2011; 471: 363.

9. Vogler $\mathrm{M}$ et al. Cell Death Differ 2009; 16: 1030

10. Ch'en IL et al. J Exp Med. 2011; 208: 633.

11. Yu L et al. Science 2004; 304: 1500.

12. Wu YT et al. Cell Death Differ 2011; 18: 26.

13. Green DR. Nat Rev Immunol 2009; 9: 353.

14. Uhl M et al. Cell Death Differ 2009; 16: 991.

15. Grivennikov SI, Greten FR, Karin M. Cell 2010; 140: 883.
} 